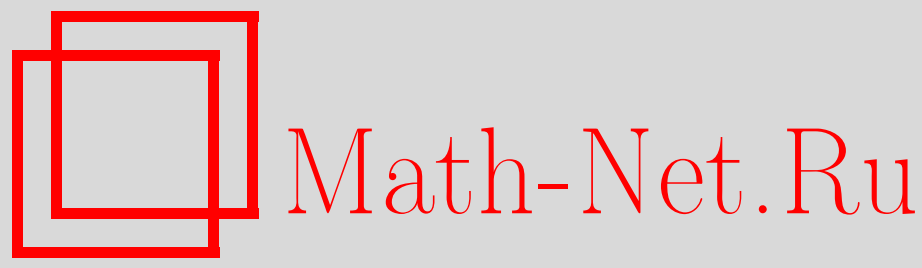

В. П. Маслов, О новых формулах распределения для классического газа, кластеров и фазовых переходов, TMФ, 2008, том 157, номер 2, 250-272

DOI: https://doi.org/10.4213/tmf6278

Использование Общероссийского математического портала Math-Net.Ru подразумевает, что вы прочитали и согласны с пользовательским соглашением http://www.mathnet.ru/rus/agreement

Параметры загрузки:

IP : 54.162 .85 .209

26 апреля 2023 г., 13:13:04

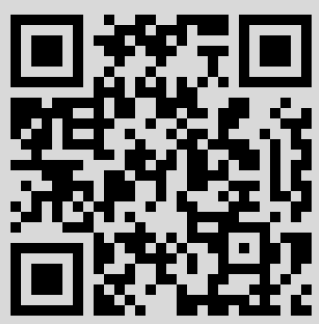




\section{О НОВЫХ ФОРМУЛАХ РАСПРЕДЕЛЕНИЯ ДЛЯ КЛАССИЧЕСКОГО ГАЗА, КЛАСТЕРОВ И ФАЗОВЫХ ПЕРЕХОДОВ}

Получено распределение типа Бозе-Эйнштейна для классического пара. Показано, что аналогом бозе-конденсата является образование кластеров. Написана новая $P V$-диаграмма для взаимодействия вида потенциала Леннарда-Джонса с использованием теории рассеяния и жесткого ограничения. Проведено сравнение с экспериментом.

Ключевые слова: кластеры, фазовые переходы, парадокс Гиббса, распределение Бозе-Эйнштейна, перколяция, нуклеация, фактор сжимаемости.

Посвящается памяти дорогого друга Толи Карацубы

\section{1. ПАРАДОКС ГИББСА КАК КОНТРПРИМЕР К РАСПРЕДЕЛЕНИЮ ГИББСА}

Каждый ученый, который опровергает теорию, устоявшуюся в течение века, рискует быть обвиненным в некомпетентности. Об этом говорит история новых открытий в физике. Таким нападкам подвергался, как известно, великий физик Больцман, который в конце концов покончил жизнь самоубийством, бросившись в пролет лестницы.

В 1900 году Планк предложил свою знаменитую формулу, описывающую излучение черного тела, которая давала результаты, совпадающие с экспериментом, но не была строго выведена. Индийский математик Бозе заметил, что фактически для вывода этой формулы необходимо использовать новую статистику, а не старую, больцмановскую. Возможно, Планк тоже знал о другой статистике, но опасался нападок или не очень верил в свой результат. Бозе, так же как и Больцман, подвергался бешеной критике, пока Эйнштейн не апробировал предложенную им статистику и философская концепция Маха ее не оправдала. В качестве аргумента против статистики Бозе физики выдвигали тот факт, что частицы, передвигаясь и меняясь местами, не могут не затрачивать энергии.

* Московский государственный университет им. М. В. Ломоносова, Москва, Россия. E-mail: v.p.maslov@mail.ru 
Различие двух статистик можно пояснить на простом примере из области финансов. Если мы хотим разместить в двух банках две копейки, то естественно считать, что у нас есть три возможности: 1) обе копейки положить в один банк; 2) обе копейки положить в другой банк; 3) одну копейку положить в один банк, другую в другой, при этом не имеет значения, какую именно копейку мы положили в тот или другой банк и какой год выпуска этих монет. Теперь рассмотрим ситуацию, при которой в банки помещаются не две копейки, а два разных бриллианта. В этом случае у нас возникают четыре возможности, так как теперь становится существенным, какой бриллиант мы положили в какой банк, а значит, вариант размещения по одному бриллианту в каждый банк "расщепляется" на два: а) бриллиант № 1 в банк № 1, бриллиант № 2 - в банк № 2; б) бриллиант № 1 - в банк № 2, бриллиант № 2 - в банк № 1 .

Ответ на недоумения физиков дала философская концепция Маха, заключающаяся в том, что исходные понятия классической физики (пространство, время, движение) субъективны по своему происхождению, а внешний мир - это "комплекс ощущений", и задача науки - описание этих субъективных ощущений. Поэтому (по Маху) если мы не можем различить частицы в нашем субъективном восприятии, то они и неразличимы.

Я предлагаю совершенно иную философию. Мы можем считать частицы как различимыми, так и неразличимыми. Это зависит только от того, в каком аспекте мы рассматриваем систему этих частиц, и от того, ответ на какой вопрос мы при этом пытаемся найти. Так, если вернуться к примеру с деньгами, людей интересует вопрос, сколько у них есть купюр определенного достоинства, а не вопрос о том, какие номера на этих купюрах. И даже, может быть, неважно, в каких они купюрах, а просто сколько денег.

Аналогичная ситуация возникает в физике. Пусть у нас имеется сосуд, наполненный газом, состоящим из множества частиц, находящихся в движении. Если мы сделаем замедленный снимок, то движущаяся частица оставит после себя некоторый след, длина которого соответствует скорости ее движения: чем быстрее движется частица, тем длиннее след остается на снимке. По такому снимку мы можем определить, сколько частиц движется в определенном интервале скоростей. И нам не важно, какая частица стоит на каком месте и какой из частиц соответствует данная скорость. Более того, для определения интервала энергии нам не важны размеры этих частиц, возможно, склеенных частично в димеры, тримеры и т.д.

Нужно вывести формулы, по которым можно определить, как распределено количество частиц по энергиям, например в каком количестве (числовом диапазоне) вероятнее всего встречаются частицы, двигающиеся с энергией, попадающей в заданный интервал. Полученные формулы оказываются распределениями типа распределения Бозе-Эйнштейна и приводят к удивительному математическому факту, который заключается в том, что существует некоторое максимальное число частиц, больше которого формулы существенно изменяются. Если число частиц много меньше указанного максимального числа, то формулы совпадают с распределением 
Гиббса с точностью до умножения на константу. Это, тем не менее, очень существенно, потому что полученная таким образом исправленная формула Гиббса уже не приводит к так называемому парадоксу Гиббса.

Парадокс Гиббса был сформулирован в работе "О равновесии гетерогенных веществ", опубликованной частями в 1876-1879 гг., и вызвал огромный интерес со стороны физиков, математиков и философов. Этой задачей занимались Пуанкаре, Лоренц, Ван-дер-Ваальс, Нернст, Планк, Ферми, Эйнштейн, фон Нейман, Шредингер, Тамм, Бриджмен, Бриллюэн, Ланде и др. С точки зрения математики парадокс Гиббса есть математический контрпример к формуле Гиббса, т.е. формула Гиббса в ее классической форме неверна и ее нужно изменить так, как предлагается ниже (см. также [1]). Это исправление ранее трактовалось как следствие квантовой теории, что неверно с математической точки зрения, поскольку переход от квантовой механики к классической не может изменить симметрии, а следовательно, и статистики.

\section{2. СОХРАНЕНИЕ СИММЕТРИИ ПРИ ПЕРЕХОДЕ ОТ КВАНТОВОЙ МЕХАНИКИ К КЛАССИЧЕСКОЙ ПРИ $H \rightarrow 0$}

Переход от квантовой механики к классической при $h \rightarrow 0$ не может менять симметрии.

В качестве примера рассмотрим спектр одномерного уравнения Шредингера с потенциальной ямой, содержащей два минимума и один локальный максимум, так называемую “яму Лифшица", симметричную относительно начала координат, в которых находится локальный максимум:

$$
-\frac{h^{2}}{2 m} \frac{d^{2}}{d x^{2}} \Psi_{n}(x)+u_{0} u\left(\frac{x}{a}\right) \Psi_{n}(x)=E_{n} \Psi_{n},
$$

где $\Psi_{n}(x)$ - собственные функции, а $E_{n}$ - собственные значения:

$$
\int_{-\infty}^{\infty} \Psi_{n}^{2}(x) d x=1,\left.\quad u(x)\right|_{x=0}=1,\left.\quad u(x)\right|_{x=-1}=\left.u(x)\right|_{x=1}=0,
$$

$a$ - некоторая характерная длина, $u_{0}$ - характерный потенциал.

Как известно, $\Psi_{n}^{2}(x)$ определяет плотность вероятности пребывания частицы в точке $x$. В силу симметрии при $E_{n}<u_{0}$ значения плотности вероятности в точках $x$ и $-x$ равны. Это означает, что вероятности пребывания частицы в обеих впадинах “ямы Лифшица" равны между собой. Этот результат, как нетрудно убедиться, сохраняется и при $h \rightarrow 0$, что противоречит классической механике в ее старом понимании, так как классическая частица не должна проникать через барьер. Для понимания этого парадокса нужно учесть, что симметрия должна быть очень точная (наноточность) и что стационарность состояния означает, что это состояние возникает в пределе бесконечно большого времени.

Абсолютно та же ситуация с тождественностью частиц - она не может исчезнуть в пределе при $h \rightarrow 0$. 
Однако если задача содержит еще один параметр, по которому надо переходить к пределу, например, как в задаче рассеяния, время ухода частицы на бесконечность, то окончательный результат может зависеть от того, в какой последовательности вычисляются пределы по этим двум параметрам.

\section{3. ПОДСЧЕТ ЧИСЛА СОБСТВЕННЫХ ЗНАЧЕНИЙ ДЛЯ УРАВНЕНИЯ ЛАПЛАСА}

Задача Дирихле для уравнения Лапласа в области $\Omega$ с нулевыми граничными условиями на границе области $\delta \Omega$

$$
\Delta u_{n}(x)+\lambda_{n} u_{n}(x)=0, \quad x \in \mathbb{R}^{3},
$$

где $\left.u_{n}(x)\right|_{\delta \Omega}=0$ для области $\Omega$, и асимптотика числа собственных значений с учетом кратностей таких, что $\lambda_{n} \leqslant \lambda$ при $\lambda \rightarrow \infty$, - давно изученная задача.

Объем области $\Omega$ обозначим через $V$. Главный член асимптотики, как известно, приближается к фазовому объему $\sqrt{\lambda^{3}} V$, где $\lambda \rightarrow \infty$. Оценка погрешности и даже следующий член асимптотики рассматривались многими математиками. Можно зафиксировать $\lambda$ и устремлять объем $V$ к бесконечности, и эта формула для числа собственных значений останется верной.

Обозначим через $\widehat{H}$ оператор Лапласа $\Delta$ с граничным условием Дирихле. Это равносильно использованию уравнения Шредингера с краевым условием в виде бесконечных стенок на границе $\delta \Omega$. Рассмотрим оператор Лапласа $\widehat{H}_{N}$ в $3 N$-мерном пространстве вида

$$
\left(\Delta_{x_{1}}+\Delta_{x_{2}}+\cdots+\Delta_{x_{N}}\right) u_{n}\left(x_{1}, x_{2}, \ldots, x_{N}\right)+\lambda_{n, N} u_{n}\left(x_{1}, x_{2}, \ldots, x_{N}\right)=0
$$

с теми же краевыми условиями по каждому $x_{i} \in \Omega: u_{n}\left(x_{1}, \ldots, x_{i}, \ldots\right)_{\delta \Omega}=0$ для симметрических по перестановкам $x_{i} \rightarrow x_{j}$ собственных функций таких, что $\lambda_{n, N} \leqslant M$, где $M \rightarrow \infty$.

Определим константу $b$ из условия

$$
\operatorname{Sp}\left(\frac{\widehat{H}}{e^{b \widehat{H}}-1}\right)=M
$$

Введем обозначение

$$
\mathbf{N}_{0}=\operatorname{Sp}\left(\frac{1}{e^{b \widehat{H}}-1}\right)
$$

Пусть $N$ в формуле (2) равно $\mathbf{N}_{0}$. Выражение вида $\sum_{i=0}^{\infty} \lambda_{i} N_{i}$ для любых $N_{i}$, удовлетворяющих условию

$$
\sum_{i=0}^{\infty} N_{i}=\mathbf{N}_{0},
$$


есть собственное значение $\widehat{H}_{\mathbf{N}_{0}}$, отвечающее симметричным по $\left(x_{i}, x_{j}\right)$ собственным функциям. Поскольку $\sum \lambda_{i} N_{i}$ является собственным значением оператора $\widehat{H}_{\mathbf{N}_{0}}$, отвечающим симметрическим собственным функциям, то сумма $\sum N_{i}$ по всевозможным $N_{i}$, удовлетворяющим соотношению (3), есть число всех собственных значений оператора $\widehat{H}_{\mathbf{N}_{0}}$, меньших $M$.

Набор чисел $\left\{N_{i}\right\}$ взаимно однозначно определяет собственную функцию оператора $\widehat{H}_{\mathbf{N}_{0}}$ через собственные функции одной частицы (1). Поэтому каждой собственной функции оператора $\widehat{H}_{\mathbf{N}_{0}}$ можно сопоставить набор чисел $\left\{N_{i}\right\}$. Эти числа называются числами заполнения, им сопоставляется число частиц на уровне энергии $\lambda_{i}$. Соответственно число $N_{i}^{(n)}=\sum_{j=i}^{n} N_{i}$ является аналогом числа заполнения для совокупности $\lambda_{i}, \lambda_{i+1}, \ldots, \lambda_{i+n}$. Мы как бы собираем эту совокупность в одну "ячейку". В теории вероятностей числа $N_{i}^{(n)}$ называются неупорядоченной выборкой $c$ возвращением из ячейки (ящика) $\left\{\lambda_{i}, \ldots, \lambda_{i+n}\right\}$. Разумеется, это свойство симметрических собственных функций несправедливо для антисимметрических собственных функций.

Пусть $P_{i}$ - проектор на собственное подпространство, отвечающее собственному значению $\lambda_{i}$, и пусть $P_{i}^{(n)}=P_{i}+P_{i+1}+\cdots+P_{i+n}$ - проектор на подпространство, порожденное $P_{i} L_{2}, P_{i+1} L_{2}, \ldots, P_{i+n} L_{2}$, где $L_{2}=L_{2}(\Omega)$ - пространство $L_{2}$ в области $\Omega$.

Пусть $n \sim \varepsilon b^{-1}$ и $\mathrm{P}$ - отношение числа собственных значений гамильтониана $\widehat{H}_{\mathbf{N}_{0}}$, отвечающих симметричным по $\left(x_{i}, x_{j}\right)$ собственным функциям, не превосходящих $M$ и удовлетворяющих неравенству

$$
\left|N_{i}^{(n)}-\operatorname{Sp}\left(P_{i}^{(n)} \frac{1}{e^{b \widehat{H}}-1}\right)\right|>\sqrt{\mathbf{N}_{0}}\left(\ln \mathbf{N}_{0}\right)^{1 / 2+\varepsilon}, \quad i=n, n+1, \ldots,
$$

где $\varepsilon>0$ - любое сколь угодно малое число, к общему числу собственных значений гамильтониана $\widehat{H}_{\mathbf{N}_{0}}$, не превосходящих $M$ и отвечающих симметрическим собственным функциям. Тогда $\mathrm{P}=O\left(\mathbf{N}_{0}^{l}\right)$, где $l$ - любое целое число.

Это пример теоремы о числе собственных значений и числе выборок [2].

Нетрудно убедиться, что $M / \mathbf{N}_{0} \rightarrow \infty$ и отвечает $\lambda \rightarrow \infty$ (или $V \rightarrow \infty$ ) в теореме Куранта.

Этот пример приведен для того, чтобы показать, что когда речь идет о числе собственных значений, то это означает их некоторую неразличимость, аналогичную неразличимости не только купюр одного достоинства, но и купюр в одну тысячу рублей относительно двух купюр по 500 рублей.

Можно, конечно, подумать, что распределение бозонного типа связано с симметрией собственных функций. Но не это главное. Главное - то, что можно использовать неупорядоченную выборку с возвращением, т.е. аддитивность $N_{i}$. В антисимметрическом случае это уже сделать невозможно.

Приведенный пример является следствием некоторых общих теорем вероятностной теории чисел [3], [4]. 


\section{4. КАКИХ ТЕОРЕМ ВЕРОЯТНОСТНОЙ ТЕОРИИ ЧИСЕЛ МОЖНО ОЖИДАТЬ}

Прежде всего на простейшем примере установим, какого типа теоремы мы можем получить. Дело в том, что в квантовой статистике, по-видимому, предполагается, что для идеального бозе-газа имеет место плотность распределения Бозе-Эйнштейна. Более того, некоторые физики полагают, что эта плотность распределения справедлива и для малого числа частиц [5].

Физики настолько привыкли к распределению Бозе-Эйнштейна, что считают, что его не нужно обосновывать и тем более не нужно точно определять фазовый переход из конденсатного состояния (бозе-конденсата) в нормальное. Но поскольку, как показал автор, общеизвестная модель финансовой пирамиды и ее обрушение сводится к задаче о фазовом переходе из конденсатного состояния в нормальное [6], то здесь уже точность и строгое обоснование оказываются совершенно необходимыми.

Испокон веков физики считают, что "лишние" частицы (сверх максимального их числа $\mathbf{N}_{0}$, т.е. при химическом потенциале, равном нулю) сосредоточиваются на нижнем уровне энергии. А где это доказано? И сколько должно быть лишних частиц, чтобы они сконденсировались на нижнем уровне? Конечно, физики понимают, что достаточно много. А что это означает по отношению к большому параметру числу частиц?

С другой стороны, известно, что существуют процессы кластеризации и перколяции, которые моделируются с помощью метода Монте-Карло. Какова физическая подоплека этих процессов? Ни тогда ли частицы начинают склеиваться в кластеры, когда "тесно", т.е. $N>\mathbf{N}_{0}$ ? В социально-экономических процессах это естественное явление [7], [8]. Если считать, что $\mathbf{N}_{0}$ - это число узлов решетки в данном объеме, то лишние частицы должны спариваться с частицами, находящимися в этих узлах, а этот процесс можно моделировать методом Монте-Карло [9], [10].

Прежде всего, чтобы понять, о каких точных математических результатах может идти речь, рассмотрим простейший пример, когда все $\lambda_{i}$ равны между собой. Именно, пусть речь идет о поведении при больших $s$ системы неотрицательных случайных величин $N_{j}, j=1, \ldots, s$, подчиненных единственному условию $\sum_{j=1}^{s} N_{j}=N$ и равномерно распределенных на множестве, задаваемом этим условием ${ }^{1)}$. Интерпретируя эти векторы как ступенчатые случайные функции, получаем следующие теоремы о слабом пределе для равномерного распределения с граничными условиями.

Теорема 1 [11]. Пусть $f_{s}(t), s=1,2, \ldots,-$ последовательность независимых неотрицательных ступенчатых случайных функций на интервале $(0,1]$ таких, что $f_{s}(t)$ постолнна на интервалах $t \in((j-1) / s, j / s], j=1, \ldots, s, \int_{0}^{1} f_{s}(t) d t=1$ u для каждого фиксированного s все функции, удовлетворяющие таким условиям, равновероятны. Тогда при $s \rightarrow \infty$ последовательность $f_{s}(t)$ слабо сходится $\kappa$

1) Целочисленность $N_{i}$ и $N$ для приводимых ниже двух теорем несущественна. 
детерминированной функици $f(t)=1$ почти наверное, т.е. для любой интегрируемой по Лебегу пробной функиии $\varphi(t)$

$$
\mathrm{P}\left(\lim _{s \rightarrow \infty} \int_{0}^{1}\left(f_{s}(t)-1\right) \varphi(t) d t=0\right)=1 .
$$

Теорема 2 [11]. Пусть $f_{s}(t), s=1,2, \ldots,-$ последовательность независимых неубывающих ступенчатых случайных функиий на отрезке $[0,1]$ таких, что $f_{s}(t)$ постоянна на интервалах $t \in((j-1) / s, j / s], j=1, \ldots, s,\left.f_{s}(t)\right|_{t=0}=0,\left.f_{s}(t)\right|_{t=1}=1$ u для каждого фиксированного s все функиии, удовлетворяющие таким условиям, равновероятны. Тогда при $s \rightarrow \infty$ последовательность $f_{s}(t)$ слабо сходится $\kappa$ детерминированной функиии $f(t)=t$ почти наверное, т.е. для любой интегрируемой по Лебегу пробной функции $\varphi(t)$

$$
\mathrm{P}\left(\lim _{s \rightarrow \infty} \int_{0}^{1}\left(f_{s}(t)-t\right) \varphi(t) d t=0\right)=1
$$

Возможно, что эти простейшие теоремы были известны и до нашей публикации [11]. Главное не это. Каждый на персональном компьютере может убедиться, что сильной сходимости эти последовательности не имеют. А это значит, что тем более невозможно доказать сходимость к плотности распределения Бозе-Эйнштейна. Можно лишь говорить о слабой сходимости [12], или о так называемом коммулятивном распределении.

\section{5. ТЕОРЕМЫ ВЕРОЯТНОСТНОЙ ТЕОРИИ ЧИСЕЛ}

Приведем теперь теоремы вероятностной теории чисел, которые нам понадобятся.

Рассмотрим всевозможные наборы целых неотрицательных чисел $N_{j k}, j=1,2, \ldots$, $k=1, \ldots, q_{i}$, удовлетворяющие условию

$$
\sum_{j=1}^{\infty} \sum_{k=1}^{q_{i}} j N_{j k} \leqslant M
$$

где

$$
q_{i}= \begin{cases}{\left[i^{d / 2}\right]} & \text { при четном } i, \\ {\left[i^{d / 2}\right]+1} & \text { при нечетном } i,\end{cases}
$$

$2<d \leqslant 3,[a]$ - целая часть числа $a, d$ - размерность (фрактальная размерность). Число этих наборов конечно, и в каждом из них есть лишь конечное число ненулевых $N_{j k}$. Для заданного набора $\left\{N_{j k}\right\}$ положим

$$
N_{j}=\sum_{k=1}^{q_{j}} N_{j k}, \quad j=1,2, \ldots, \quad N_{j}^{n}=\sum_{i=j}^{j+n} N_{i}, \quad N=\sum_{j=1}^{\infty} N_{j} .
$$

TEOPEMA 3 [13]. Пyсms

$$
j \geqslant C b^{-\gamma} \text { для некоторьх } \gamma \in(0,1], \quad C>0,
$$


и пусть $\chi(x)$ - растущая на бесконечности положительная функиия. Тогда существуют такие постоянные $C_{m}$, что выполнены оценки

$$
\mathrm{P}\left(\left|N_{j}^{(n)}-b^{-d / 2} \int_{b j}^{b(j+n)} \frac{x^{d / 2-1} d x}{e^{x}-1}\right|>N_{0}^{\alpha} \sqrt{\ln N_{0}} \chi\left(N_{0}\right)\right) \leqslant C_{m} N_{0}^{-m},
$$

где $m=0,1,2, \ldots$,

$$
\alpha=\frac{\gamma}{2}+2 \frac{1-\gamma}{d}, \quad N_{0}=M^{(d / 2) /(d / 2+1)} \frac{\Gamma(d / 2) \zeta(d / 2)}{(\Gamma(d / 2+1) \zeta(d / 2+1))^{(d / 2) /(d / 2+1)}},
$$

$\Gamma(x)$ - гамма-функция, $(x)$ - дзета-функция Римана.

При дополнительном условии на интервал $[j, j+n]$ (он не должен быть слишком узким) эта оценка не может быть улучшена. Этот последний факт - самый важный.

\section{6. УЧЕТ ХИМИЧЕСКОГО ПОТЕНЦИАЛА}

Пусть $M$ и $N$ - целые положительные числа. Рассмотрим множество всевозможных наборов $\left\{N_{j}\right\}$ целых неотрицательных чисел $N_{j}, j=1,2, \ldots$, таких, что выполнены соотношения

$$
\sum_{j=1}^{\infty} N_{j}=N, \quad \sum_{j=1}^{\infty} j N_{j} \leqslant M
$$

При выполнении определенных условий, когда $M$ и $N$ стремятся к бесконечности, большинство (в смысле вышеприведенной вероятностной меры Р) наборов $\left\{N_{j}\right\}$ концентрируется вблизи некоторого предельного распределения. Точнее говоря, суммы $\sum_{j=1}^{\infty} N_{j}$ с большой вероятностью мало отличаются от соответствующих кумулятивных интегралов для предельного распределения.

Рассмотрим систему уравнений

$$
\sum_{j=1}^{\infty} \frac{j^{d / 2-1}}{e^{b j+\mu}-1}=N, \quad \sum_{j=1}^{\infty} \frac{j^{d / 2}}{e^{b j+\mu}-1}=M
$$

Эта система имеет единственное вещественное решение $(b, \mu)$ такое, что $b>0$ и $\mu>0,1 \leqslant d \leqslant 3$.

ТЕОРема 4 [14]. Пусть выполняется условие (7) и пусть ұ(x)-растущая на бесконечности положительная функция. Тогда существуют такие постоянные $C_{m}$, что выполнены оценки

$$
\mathrm{P}\left(\left|\sum_{i=j}^{\infty} N_{i}-b^{-d / 2} \int_{b j}^{\infty} \frac{x^{d / 2-1} d x}{e^{x+\mu}-1}\right|>N^{\gamma / 2+2(1-\gamma) / d} \sqrt{\ln N} \chi(N)\right) \leqslant C_{m} N^{-m}
$$

где $m=0,1,2 \ldots$.

4 Теоретическая и математическая физика, т. 157, № 2, 2008 г. 


\section{7. СЛЕДСТВИЯ ТЕОРЕМ 3 И 4. КЛАСТЕРИЗАЦИЯ}

Выведем формулы, по которым можно определить, как распределены частицы по энергиям, например в каком количестве (числовом диапазоне) вероятнее всего встречаются частицы, двигающиеся с энергиями, попадающими в заданный интервал [15].

Пусть $N \rightarrow \infty$ - число невзаимодействующих частиц с гамильтонианом $p^{2} / 2 m$ в объеме $V, p=\left(p_{x}, p_{y}, p_{z}\right)$ - импульс. Разобьем пространство импульсов $p$ на большое число областей

$$
\begin{gathered}
E_{l} \leqslant \frac{p^{2}}{2 m} \leqslant E_{l+1}, \quad l=0, \ldots, s-1, \quad s \rightarrow \infty \\
\Delta E=E_{l+1}-E_{l}=E_{0}>0, \quad p \in \mathbb{R}^{3},
\end{gathered}
$$

где $E_{0}$ - некоторое конечное число (фиксированный интервал). Тогда $E_{l}=E_{0}(l+1)$. Пусть задано достаточно большое число $M$ (уровень энергии). Пусть $N_{l}$ - неупорядоченная выборка с возвращением в ячейку $E_{l+1}-E_{l}$. Иными словами, неупорядоченная выборка с возвращением из $N$ шаров по ячейкам (“энергетическим ящикам")

$$
V \int_{E_{l}}^{E_{l+1}} \frac{p^{2}}{2 m} d p=\frac{2}{5}(2 m)^{3 / 2}\left(E_{l+1}^{5 / 2}-E_{l}^{5 / 2}\right) V
$$

приводит к соотношению

$$
\sum_{l=1}^{s} N_{l} E_{l} q_{l} \leqslant M, \quad q_{l}=\left\{\begin{array}{ll}
{\left[l^{3 / 2}\right]} & \text { при четном } l, \\
{\left[l^{3 / 2}+1\right]} & \text { при нечетном } l,
\end{array} \quad s \rightarrow \infty, \quad M \rightarrow \infty .\right.
$$

Это значит, что для кратностей вида $q_{l}$ и чисел заполнения $N_{l}$, или неупорядоченной выборки с возвращением, выполняется теорема 4.

Положим $\Delta N_{l}=\mathbf{N}_{0}^{\alpha_{l}+\varepsilon}$, где $\varepsilon$ - достаточно малое положительное число, $1 / 2<$ $\alpha_{l}<2 / 3$ зависит от $l$ и вычисляется для $d=3$ в теореме $3, \mathbf{N}_{0}$ - число частиц в перенасыщенном газе (см. ниже). В силу того, что плотность $\rho_{E_{l}}=\sum_{j=l}^{s} N_{j} / \mathbf{N}_{0}$, после применения формул Эйлера-Маклорена к ряду

$$
\sum_{j=l}^{s} \bar{N}_{j}=\sum_{j=l}^{s} q_{j} \frac{1}{e^{b j}-1}
$$

получим

$$
\sum_{j=l}^{s} q_{j} \frac{1}{e^{b j}-1} \sim \int_{l}^{\infty} \frac{x^{1 / 2}}{e^{b x}-1} d x
$$

Отсюда, сделав замену $x=p^{2} / 2 m$ и поделив $(14),(15)$ на $\mathbf{N}_{0}$, получим утверждение следующей теоремы, являющейся следствием теоремы 3 при $d=3$ [13].

ТеОРема 5. Имеет место соотношение

$$
\mathrm{P}\left(\left|\rho_{E_{l}}-\int_{p^{2} / 2 m \geqslant E_{l}}\left(e^{b p^{2} / 2 m}-1\right)^{-1} d p / \int\left(e^{b p^{2} / 2 m}-1\right)^{-1} d p\right| \geqslant \mathbf{N}_{0}^{\alpha_{l}-1}\right)=O\left(\mathbf{N}_{0}^{-k}\right),
$$


¿əe

$$
\mathbf{N}_{0}=\frac{1}{\left(m E_{0}\right)^{3 / 2}} \int\left(e^{-b p^{2} / 2 m}-1\right) d p,
$$

$k$ - любое целое число, $\varepsilon>0$ - сколь угодно малое число, и при $E_{l} \sim E_{0} \mathbf{N}_{0}^{\gamma_{l}}, 0<$ $\gamma_{l} \leqslant 2 / 3$, имеем $\alpha_{l}=2 / 3-\gamma_{l} / 4$. Здесь $\mathrm{P}$ есть лебегова мера фазового обгема, определенного в скобках (16), относительно всего объема (см. [1]).

Можно интерпретировать $\mathbf{N}_{0}$ как максимальное число частиц, а именно как число частиц в максимально перенасыщенном паре. Пусть мы добавили в сосуд объема $V \quad N-\mathbf{N}_{0}$ "лишних" частиц, при этом $N$ превышает $\mathbf{N}_{0}$ настолько, что $N-\mathbf{N}_{0} \cong \mathbf{N}_{0}^{1 / 2+\kappa}$, где $\kappa<1 / 6$, тогда лишние частицы сосредоточатся на энергиях, не превышающих

$$
E_{l} \sim E_{0} N^{\gamma_{l}}, \quad \gamma_{l}=4\left(\frac{1}{6}-\kappa\right) .
$$

Ниже этих энергий распределение (16) (типа Бозе-Эйнштейна) несправедливо.

Лишние частицы $N-\mathbf{N}_{0}$ можно интерпертировать как число кластеров (димеров). В результате получается фазовый переход первого рода. Действительно, как уже говорилось выше, только те частицы, которые движутся с энергией, превышающей энергию лишних частиц (17), можно с уверенностью считать частицами "чистого" газа (пара), а остальные, возможно, кластеризовались или перемешались с кластеризованными.

Известно такое понятие, как сжимаемость газов. В учебнике по молекулярной физике [16] (с. 50-51) поведение фактора сжимаемости газа $Z=P V / R T N$, где $P$ давление, $R$ - постоянная Больцмана, $T$ - температура, $N$ - число частиц, при изотермическом увеличении давления иллюстрируется с помощью усредненных диаграмм Хоугена и Ватсона, которые были построены для семи газов $\left(\mathrm{H}_{2}, \mathrm{~N}_{2}, \mathrm{CO}\right.$, $\left.\mathrm{NH}_{3}, \mathrm{CH}_{4}, \mathrm{C}_{3} \mathrm{H}_{8}, \mathrm{C}_{3} \mathrm{H}_{12}\right)$. Диаграмма показывает, что при низких температурах фактор сжимаемости сначала уменьшается, претерпевая резкий скачок вниз при значении 0.5, и лишь затем устремляется вверх, возвращается к исходному значению, а затем и превышает его. Объяснение этому явлению дается лишь для газа Ван-дер-Ваальса для вириального разложения при условии $|1-Z| \ll 1$, а поскольку минимальный фактор сжимаемости равен 0.2 , это явление с помощью обычной классической теории газа объяснить нельзя.

Пусть $T$ - температура насыщенного пара в объеме $\Omega$. Понижаем температуру до $T_{0}$ так, чтобы

$$
\frac{R\left(T-T_{0}\right)}{E_{0}}=c\left(\frac{R T_{0}}{E_{0}}\right)^{s},
$$

где $1 / 4<s \leqslant 1 / 2, c$ - константа. Тогда конденсат возникает при (см. [1])

$$
E_{1} \cong c E_{0}\left(\frac{R T_{0}}{E_{0}}\right)^{2-4 s}
$$

Отсюда получаем число частиц перенасыщенного пара при температуре $T_{0}$

$$
N_{0}=\int_{0}^{\infty} \frac{1}{e^{\beta_{0} p^{2} / 2 m}-1} d p,
$$


где $\beta_{0}=\left(R T_{0}\right)^{-1}$, число лишних (добавленных) частиц

$$
\int_{0}^{\infty} \frac{1}{e^{\beta p^{2} / 2 m}-1} d p-N_{0},
$$

где $\beta=(R T)^{-1}$. Однако число частиц после окончательного выпадения в конденсат, т.е. число частиц вне конденсата (число частиц насыщенного пара), равно

$$
\int_{\left(R T_{0}\right)^{-s}}^{\infty} \frac{1}{e^{\beta_{0} p^{2} / 2 m}-1} d p,
$$

а значит, меньше, чем за вычетом лишних частиц. Таким образом, происходит скачок числа частиц, т.е. фазовый переход 1-го рода, в отличие от перехода к перенасыщенному пару (фазовый переход 3-го рода). Значит, метастабильное состояние перенасыщенного пара, когда только начинают появляться кластеры, постепенно перейдет в стабильное состояние насыщенного пара со скачком числа частиц и энтропии $[1]^{2)}$.

Средняя энергия и сжимаемость не зависят от массы частицы. Это легче всего проверить, если параметр $b$ безразмерный.

Таким образом, если все частицы склеились в димеры, то число частиц уменьшилось в два раза. А это значит, что химический потенциал в теореме 4 увеличивается. Поскольку при уменьшении температуры, как это следует из вышесказанного, возникает явление, похожее на бозе-конденсат, которое в нашем случае соответствует возникновению кластеров, что приводит к увеличению сжимаемости, то и наоборот, при увеличении температуры число кластеров будет уменьшаться. Этот факт хорошо известен экспериментаторам.

\section{8. ОБЩЕЕ РАСПРЕДЕЛЕНИЕ, ПЕРЕХОДЯЩЕЕ В РАСПРЕДЕЛЕНИЕ ГИББСА}

Мы будем рассматривать достаточно общую классическую функцию Гамильтона $H(p, q)$ и сделаем относительно нее три предположения.

Пусть $H(p, q)$, где $q \in \mathbb{R}^{3}, p \in \mathbb{R}^{3}$, - достаточно гладкая функция такая, что:

1) $H(p, q) \rightarrow \infty$ при $|p|+|q| \rightarrow \infty$ не медленнее, чем $(|p|+|q|)^{\alpha}$ при некотором $\alpha>0$

2) интеграл $\int_{0}^{A}(H(p, q))^{-1} d p d q$ сходится для любого фиксированного $A, p_{i} \leqslant A$, $q_{i} \leqslant A ; i=1,2,3$;

$3)$ производная $V^{\prime}(\Lambda)$ функции

$$
V(\Lambda)=\int_{H(p, q) \leqslant \Lambda} d p d q
$$

${ }^{2)}$ В учебнике [17] (с. 199, сноска) сказано: "Явление накапливания частиц в состоянии с $\varepsilon=0$ часто называют "конденсацией Бозе-Эйнштейна". Подчеркнем, что речь может идти при этом разве что о "конденсации в импульсном пространстве"; никакой реальной конденсации в газе, конечно, не происходит" (т.е. в квантовом идеальном газе, например в гелии). В то время, когда был написан этот учебник, разумеется, не были известны эксперименты по гелию [18] (см. также [19], [20]). В последней из указанных работ используется метод Монте-Карло, как в теории перколяции [21]. 
- достаточно гладкая функция.

Пусть $E_{0}$ - некоторое значение энергии, много меньшее средней энергии молекул газа. Пусть имеется $N$ не взаимодействующих между собой частиц, каждая из которых описывается гамильтонианом $H(p, q)$, и пусть энергия $N$ частиц ограничена величиной $M \gg E_{0}$, т.е. $M / E_{0} \rightarrow \infty$. Определим величину $b$ из соотношения

$$
\int H(p, q)\left(e^{b H(p, q)}-1\right)^{-1} d p d q=M, \quad b \rightarrow 0 \quad \text { при } \quad M \rightarrow \infty .
$$

Введем число

$$
\mathbf{N}_{0}=\frac{1}{\left(m E_{0}\right)^{3 / 2}} \int\left(e^{b H(p, q)}-1\right)^{-1} d p d q,
$$

которое мы будем называть числом частиц, насыщающих объем $\Omega$, и предположим, что число частиц $N \leqslant \mathbf{N}_{0}$.

Подобно тому, как это было сделано выше, разобьем фазовое пространство $p, q$ на конечное число областей:

$$
\begin{gathered}
E_{l} \leqslant H(p, q) \leqslant E_{l+1}, \quad l=0, \ldots, s-1, \quad s \rightarrow \infty, \\
\Delta E=E_{l+1}-E_{l}=E_{0}>0, \quad p \in \mathbb{R}^{3}, \quad q \in \mathbb{R}^{3} .
\end{gathered}
$$

Далее, аналогично предыдущему, произведем неупорядоченную выборку с возвращением $N_{l}$ из $N$ частиц в "ящик" $E_{l} \leqslant H(p, q) \leqslant E_{l+1}$ при условии

$$
\sum_{l=1}^{s} N_{l} E_{l} q_{l} \leqslant M, \quad q_{l}=\left\{\begin{array}{ll}
{\left[V^{\prime}(l)\right]} & \text { при четном } l, \\
{\left[V^{\prime}(l)+1\right]} & \text { при нечетном } l,
\end{array} \quad M \rightarrow \infty, \quad s \rightarrow \infty .\right.
$$

Обозначим через $\rho_{E_{l}}-$ плотность числа частиц в интервале, большем или равном $E_{l}$, а именно $\rho_{E_{l}}=\sum_{j=l}^{\infty} N_{j} / \mathbf{N}_{0}$.

Приведем теорему, аналогичную теореме 5. Здесь Р означает меру фазового объема, определенного в скобках (16), относительно всего объема (19) [14].

Теорема 6. Имеет место соотношение

$$
\mathrm{P}\left(\left|\rho_{E_{l}}-\int_{H(p, q) \geqslant c / b}\left(e^{b H(p, q)}-1\right)^{-1} d p d q / \int\left(e^{b H(p, q)}-1\right)^{-1} d p d q\right| \geqslant N^{-1 / 2+\varepsilon}\right)=O\left(\mathbf{N}_{0}^{-k}\right),
$$

где $k$ - любое челое число, $c>\varepsilon, \varepsilon-$ - коль угодно малое, не зависящее от $b$ число.

ДокАзАтельство. Теорема следует из общей теоремы 3 о бозе-распределении. Выберем такое разбиение, что $E_{l+1}-E_{l}=E_{0}$ и $\left(E_{l+1}-E_{l}\right) / E>\mathbf{N}_{0}^{-1 / 4}$. Тогда $E_{l}=E_{0}(l+1)$.

Пусть $N_{l}$ - неупорядоченная выборка с возвращением в ячейку $E_{l+1}-E_{l}$. Неупорядоченная выборка с возвращением из $N$ шаров по ячейкам

$$
\int_{E_{l}}^{E_{l+1}} H(p, q) d p d q=\int_{E_{0} l}^{E_{0}(l+1)} \lambda V^{\prime}(\lambda) d \lambda
$$


приводит к соотношению

$$
\sum_{l} N_{l} E_{l} q_{l} \leqslant M, \quad q_{l} \cong C\left[V^{\prime}\left(E_{0} l\right)\right]
$$

где $C$ - константа. Это значит, что для кратностей вида $\left[V^{\prime}\left(E_{0} l\right)\right]$ и чисел заполнения $N_{l}$ выполняется теорема из работы [3]. А именно, положим $\Delta N=\mathbf{N}_{0}^{1 / 2+\varepsilon}$, где $\varepsilon$ - достаточно малое положительное число. Существуют такие постоянные $C_{k}$, $k=1,2, \ldots$, что для любого $l \in \mathbb{Z}_{+}$справедлива оценка

$$
\mathrm{P}\left(\left|\sum_{l=1}^{s}\left(N_{l}-\bar{N}_{l}\right)\right|>\Delta N\right) \leqslant C_{k} \mathbf{N}_{0}^{-k}
$$

где $\bar{N}_{l}=q_{l} /\left(e^{b l}-1\right), l=1,2, \ldots$, и в нашем случае $q_{l}=\left[V^{\prime}\left(E_{0} l\right)\right]$.

Поскольку по определению $\rho_{E_{l}}=\sum_{j=1}^{l} N_{j} / \mathbf{N}_{0}$, то отсюда следует, что после применения формул Эйлера-Маклорена к ряду

$$
\sum_{l=l_{0}}^{s} \bar{N}_{l}=\sum_{l=l_{0}}^{s} q_{l} \frac{1}{e^{b l}-1}
$$

мы можем перейти от ряда к интегралу, после чего, поделив (21) на $\mathbf{N}_{0}$, получим утверждение теоремы аналогично [1].

Вычислим константы $b$ и $c$ как функции от $M$ и $N$ исходя из следующих соотношений:

$$
\begin{gathered}
\int H(p, q)\left(c e^{b H(p, q)}-1\right)^{-1} d p d q=M, \\
\int\left(c e^{H(p, q)}-1\right)^{-1} d p d q=N .
\end{gathered}
$$

Справедлива следующая теорема.

Теорема 7. Имеет место соотношение

$$
\begin{gathered}
\mathrm{P}\left(\left|\rho_{E_{l}}-\int_{H(p, q) \geqslant E_{l}}\left(c e^{b H(p, q)}-1\right)^{-1} d p d q / \int\left(c e^{b H(p, q)}-1\right)^{-1} d p d q\right| \geqslant\right. \\
\left.\geqslant \sqrt{\frac{\ln N}{N}} \chi(N)\right)=O\left(N^{-k}\right),
\end{gathered}
$$

где $k$ - любое иелое число, $E_{l} \geqslant \varepsilon / b, \varepsilon>0$ (здесь $c=e^{-\mu b}$, где $\mu \leqslant 0$ - химический потенииал).

Доказательство аналогично доказательству теоремы 6.

Мы обозначим распределение вероятности (24) отвечающее гамильтониану $H(p, q)$, через $\mathrm{P}(H(p, q))$. 


\section{9. УЧЕТ ВЗАИМОДЕЙСТВИЯ МЕЖДУ ЧАСТИЦАМИ}

Рассмотрим взаимодействие частиц, описываемое потенциалом Леннарда-Джонса

$$
\Phi(r)=4 \varepsilon\left\{\left(\frac{a}{r}\right)^{12}-\left(\frac{a}{r}\right)^{6}\right\},
$$

где $\varepsilon$ и $a$ - постоянные, имеющие размерность энергии и длины соответственно, $r^{2}=$ $\left(x_{i}-x_{j}\right)^{2}+\left(y_{i}-y_{j}\right)^{2}+\left(z_{i}-z_{j}\right)^{2}, i \neq j, x_{i}, y_{i}, z_{i}$ - координаты $i$-й частицы. Длина $a$ есть эффективный радиус частицы, при $r=a$ имеем $\Phi(a)=0$. Величина $\varepsilon$ определяет глубину потенциальной ямы $\Phi(r)$ и, следовательно, характеризует интенсивность межмолекулярных сил.

Обычное уравнение для одетого потенциала [22] должно иметь вид

$$
u(x)=\int \Phi(x-\xi) \operatorname{Li}_{3 / 2}\left(e^{-b u(\xi)}\right) d \xi
$$

где $\Phi(x-\xi)$ - потенциал $(25), \mathrm{Li}_{3 / 2}$ - полилогарифм. Его можно переписать, подставив вместо ядра интегрального оператора псевдодифференциальный оператор $\widetilde{\Phi}(i a(\partial / \partial x))$, где $\widetilde{\Phi}$ - преобразование Фурье от потенциала взаимодействия. В нулевом приближении при $a \rightarrow 0$ получается, что $\widetilde{\Phi}(0)$ есть интеграл от потенциала $(25)$ по $r$ от некоторого $r_{0}$ до $\infty$. Этот интеграл существенно зависит от $r_{0}$. Как выбрать $r_{0}$ ?

Рассмотрим рассеяние одной частицы на другой [23], [24]. Предположим, что при $t \rightarrow-\infty$ скорости двух сталкивающихся частиц равны $\mathbf{v}_{1}^{\text {in }}, \mathbf{v}_{2}^{\text {in }}$. Это означает, что при $t=-\infty$ траектории частиц приближаются к прямым линиям. В терминах переменной $r=r_{2}-r_{1}$ при $t \rightarrow-\infty$ радиус-вектор $r(t)$ асимптотически приближается к функции $r^{\text {in }}=\rho+\mathbf{v}^{\text {in }} t$, где $\rho \mathbf{v}^{\text {in }}=0, \mathbf{v}^{\text {in }}=\mathbf{v}_{2}^{\text {in }}-\mathbf{v}_{1}^{\text {in }}$. Постоянный вектор $\rho$ называют прицельным параметром. Величина $\rho$ равна расстоянию между прямыми линиями, по которым двигались бы частицы в отсутствие взаимодействия. После столкновения при $t \rightarrow \infty$ скорости частиц равны $\mathbf{v}_{1}^{\text {out }}, \mathbf{v}_{2}^{\text {out }}$. Это означает, что радиус-вектор $r(t)$ асимптотически приближается к функции $r^{\text {out }}=\mathbf{n}+\mathbf{v}^{\text {out }} t$. Траектории $r^{\text {in }}(t)$ и $r^{\text {out }}(t)$, являющиеся прямыми линиями, называются входящими и выходящими асимптотами. Величина относительной скорости в in- и out-состояниях сохраняется: $\left|\mathbf{v}^{\text {in }}\right|=\left|\mathbf{v}^{\text {out }}\right|=v$.

Момент и энергия при рассеянии двух частиц равны соответственно

$$
M_{0}=\frac{m}{2}[\rho \mathbf{v}], \quad E=\frac{m}{4} v^{2} .
$$

На рис. 1 изображены входящие и выходящие асимптоты в случае потенциала Леннарда-Джонса. Значению $\rho=\mathbf{b}_{1}$ соответствует отталкивание, а значению $\rho=$ $\mathbf{b}_{2}$ - притяжение. Условие на точку поворота $r_{0}$ имеет вид

$$
\frac{m v^{2}}{4}-\frac{m(\rho v)^{2}}{2 r^{2}}-\Phi(r)=0, \quad r=r_{0} .
$$




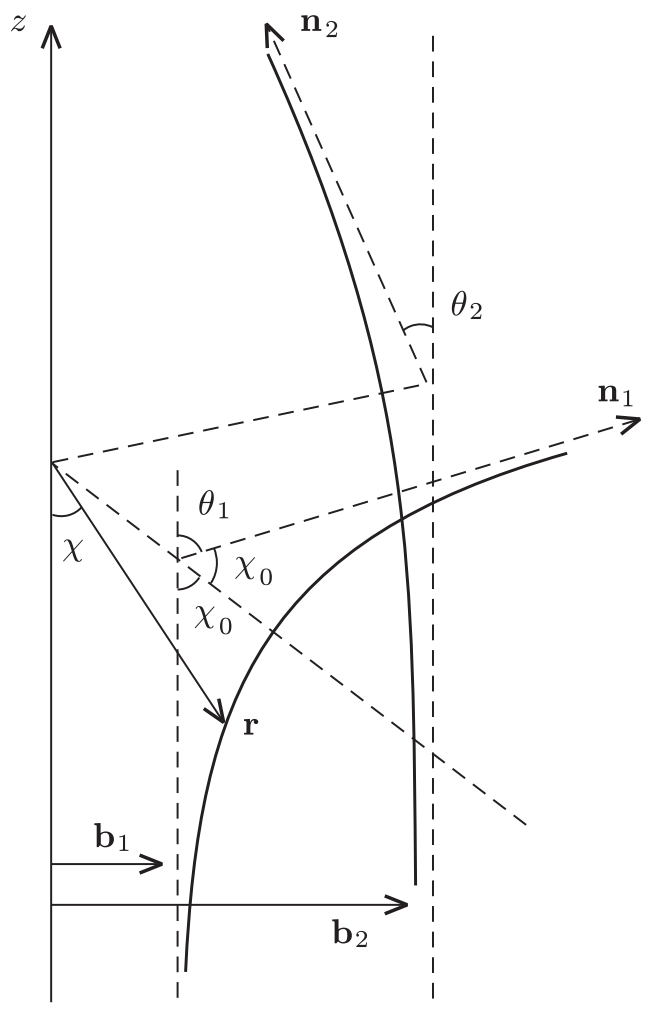

Рис. 1. Рассеяние в случае потенциала Леннарда-Джонса.

Вычислим значение $\widetilde{\Phi}(0)$ для потенциала $(25)$, зависящее от $r_{0}$ :

$$
\begin{aligned}
\widetilde{\Phi}(0) & =N \int_{r_{0}}^{\infty} \Phi(|r|) d r=4 \varepsilon N \int_{r_{0}}^{\infty}\left(\frac{a^{12}}{r^{12}}-\frac{a^{6}}{r^{6}}\right) d x d y d z= \\
& =\frac{16}{3} \pi \varepsilon a^{3} N \int_{r / a}^{\infty}\left(\frac{1}{\xi^{10}}-\frac{1}{\xi^{4}}\right) d \xi= \\
& =\frac{16 \pi}{3} \varepsilon a^{3} N\left\{-\frac{1}{9}\left(\frac{a}{r_{0}}\right)^{9}+\frac{1}{3}\left(\frac{a}{r_{0}}\right)^{3}\right\}= \\
& =\frac{16}{3} \pi \varepsilon a^{3} N\left(-\frac{1}{3} \frac{a^{3}}{r_{0}^{3}}+\frac{1}{9} \frac{a^{9}}{r_{0}^{9}}\right),
\end{aligned}
$$

где $r / a=\xi$. В частности, при $r_{0}=a \widetilde{\Phi}(0)=-8 \varepsilon a^{3} \alpha / 9, \alpha=4 \pi / 3 ;$ при $r_{0}=a / \sqrt[6]{3}$ $\widetilde{\Phi}(0)=0$.

В нанотрубке прицельный параметр $\rho$ можно считать равным нулю. В этом случае находим значение $r_{0}$ :

$$
\xi^{12}-\xi^{6}=\frac{p^{2}}{4 \varepsilon m}, \quad \xi=\frac{a}{r_{0}}, \quad \xi^{6}=x, \quad x^{2}-x-\frac{p^{2}}{4 \varepsilon m}=0
$$




$$
\begin{gathered}
x_{1,2}=\frac{1}{2}+\sqrt{\frac{1}{4}+\frac{p^{2}}{4 \varepsilon m}=\frac{1}{2}+\frac{1}{2} \sqrt{1+\frac{p^{2}}{\varepsilon m}},} \\
\frac{a}{r_{0}}=\left[\frac{1}{2}\left(1+\sqrt{1+\frac{p^{2}}{\varepsilon m}}\right)\right]^{1 / 6},
\end{gathered}
$$

т.е. при $p=0$ получаем $r_{0}=a$.

Следовательно, мы не можем пользоваться соотношением (26) для одетого потенциала, поскольку при таком выборе $r_{0}$ потенциал взаимодействия Леннарда-Джонса зависит от разности импульсов частиц: $\Phi\left(r, r_{0}\right)=\Phi\left(r, r_{0}(|p|)\right)$. Теперь потенциал Леннарда-Джонса может быть записан в виде $\Phi\left(r_{0}, r\right)=\Phi\left(r_{0}(p), r\right)$, а следовательно, поскольку $|p|=\left|p_{i}-p_{j}\right|$, уравнение для одетого потенциала будет выглядеть следующим образом:

$$
u(p, x)=N \iint \Phi\left(r_{0}(p-\eta),|x-\xi|\right) \frac{d \eta d \xi}{e^{b\left(\eta^{2} / 2 m+u(\eta, \xi)\right)}-1}, \quad p \in \mathbb{R}^{3}, \quad x \in \mathbb{R}^{3} .
$$

Поскольку нет внешнего потенциала и распределение зависит от $x$ только через одетый потенциал, то можно положить, что $u(p, x)=u(p)$, т.е. не зависит от $x$.

Сделав замену $x-\xi=y$ и проинтегрировав по $y$ от $r_{0}$ до $\infty$, получим

$$
u(p)=\frac{16}{3} \pi \varepsilon a^{3} N \int \frac{\frac{1}{9}\left[\frac{1}{2}+\frac{1}{2} \sqrt{1+\frac{(p-\eta)^{2}}{\varepsilon m}}\right]^{3 / 2}-\frac{1}{3}\left[\frac{1}{2}+\frac{1}{2} \sqrt{1+\frac{(p-\eta)^{2}}{\varepsilon m}}\right]^{1 / 2}}{e^{b\left\{\eta^{2} / 2 m-\mu+u(\eta)\right\}}-1} d \eta .
$$

Здесь $\mu \leqslant 0$ - химический потенциал, он равен нулю при $T=T_{\text {кр }}, P=P_{\text {кр }}$, только

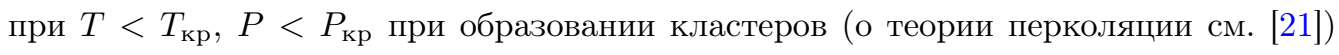
химический потенциал возрастает по модулю.

9.1. Общий случай. Учтем прицельный параметр $\rho$ в общем случае.

В случае классической задачи рассеяния с потенциалом типа Леннарда-Джонса зависимость $r_{0}(E, \theta)$ находим из условия

$$
1-\frac{\rho^{2}}{r_{0}}-\frac{\Phi\left(r_{0}\right)}{E}=0
$$

Через $\mathrm{P}_{\rho}\left(\left(p_{1}-p_{2}\right)^{2} / 2\right)$ при фиксированном $\rho$ мы определим одномерное распределение разности импульсов в задаче рассеяния:

$$
\mathrm{P}_{\rho}\left(\frac{\left(p_{1}-p_{2}\right)^{2}}{m}\right)=\frac{1}{e^{b^{\prime}\left(p_{1}-p_{2}\right)^{2} / m-\mu^{\prime} b^{\prime}}-1}\left(\int_{p_{\min }}^{\infty} \frac{d \zeta}{e^{b^{\prime} \zeta^{2} / m-\mu^{\prime} b^{\prime}}-1}\right)^{-1},
$$

где $p_{\min }=p_{\min }(\rho)$ при $r_{0} \sim a$ определяется через минимальную энергию задачи рассеяния при фиксированном параметре $\rho$, например для $\rho>2 a, r_{0}<\rho$

$$
E_{\min }=\min E=4 \varepsilon a^{6} \max _{r_{o}<\rho} \frac{1}{r_{0}^{6}}\left(\frac{\rho^{2}}{r_{0}^{2}}-1\right)^{-1}=27 \varepsilon \frac{a^{6}}{\rho^{6}} .
$$


Отсюда

$$
p_{\min }=\sqrt{m E_{\min }} \quad \text { и } \quad r_{0}=\sqrt{\frac{2}{3}} \rho .
$$

Поскольку

$$
\Phi(r) \sim-4 \varepsilon \frac{a^{6}}{r^{6}}
$$

то мы получаем

$$
\frac{\Phi(r)}{E_{\min }} \sim-\frac{4}{27} \frac{\rho^{6}}{r^{6}} .
$$

Пусть $E_{0}-$ универсальная энергия (13). Находим $b^{\prime}$ из соотношения

$$
\frac{1}{\sqrt{m E_{0}}} \int_{p_{\min }}^{\infty} \frac{d p}{e^{b^{\prime} p^{2} / m-\mu^{\prime} b}-1}=\left(\frac{N}{2}\right)^{1 / 3} .
$$

Отсюда имеем $b^{\prime} \ll b$.

Полагая $p_{1}^{\prime}=p+1 / \sqrt{b}, p_{2}^{\prime}=p_{2} / \sqrt{b}$, легко видеть, что распределение $\mathrm{P}_{\rho}\left(\left(p_{1}-\right.\right.$ $\left.p_{2}\right)^{2} / m$ ) для больших $p_{1}^{\prime}$ и $p_{2}^{\prime}$ стремится к $\delta$-функции и его максимум достигается в точке $\left(p_{1}-p_{2}\right)_{\min }$.

9.2. Уравнение для одетого потенциала. В задаче многих тел, как правило, считается, что потенциал взаимодействия зависит только от координат. Однако в модельном потенциале Бардина и во многих задачах теории твердого тела потенциал взаимодействия зависит также от импульсов. В настоящей работе мы хотим отметить следующее важное обстоятельство. Если в квантовой теории многих тел это правило действительно можно применить, то в задачах классической механики нужно существенно изменить потенциал взаимодействия даже двух частиц. При таком взаимодействии мы сводим задачу к задаче для одной частицы во внешнем поле, образованном другой частицей, а следовательно, внешний потенциал воздействует на частицу только до точки поворота, которая зависит от величины энергии и момента в радиально-симметричном случае. Если мы учтем это обстоятельство, то "реальный” потенциал взаимодействия уже будет существенно зависеть от импульсов частиц. Во всех задачах с самосогласованным (средним) полем этот факт дает существенную коррекцию.

Когда мы говорим, что вероятность распределения для второй частицы та же, что и для первой, а это есть принцип самосогласованного поля, мы обязаны учесть, что вероятность того, что частицы находятся на расстоянии, меньшем $r_{0}$, равна нулю. Иначе говоря, плотность распределения второй частицы должна быть умножена на тета-функцию, которая равна нулю, если вторая частица отстоит от первой на расстояние, меньшее $r_{0}$. Только тогда мы можем сделать предположение, что вторая частица распределена так же, как первая. В квантовой теории это существенно лишь в задачах, в которых потенциал взаимодействия имеет столь большую сингулярность, что ее приходится регуляризовать с помощью задачи двух тел (задача рассеяния). Это замечание сделал Л. Д. Ландау на докладе Н. Н. Боголюбова о слабонеидеальном бозе-газе и в дальнейшем воплотил в жизнь С. Т. Беляев. Если в этой 
задаче мы рассмотрим квазиклассическую асимптотику при $h \rightarrow 0$, получим именно соотношение, которое учитывает $r_{0}$.

Окончательно для одетого потенциала с учетом жесткого ограничения уравнение выглядит следующим образом:

$$
u(p, \rho)=\frac{16}{3} \pi \varepsilon a^{3} N \iint_{0}^{2 \pi}\left(\frac{1}{9} \frac{a^{9}}{r_{0}^{9}}-\frac{1}{3} \frac{a^{3}}{r_{0}^{3}}\right) \frac{1}{e^{b\left(\left(p^{\prime}\right)^{2} / 2 m+u\left(p^{\prime}, \rho\right)-\mu\right)}}\left(p^{\prime}\right)^{2} d p^{\prime} d \phi,
$$

где $r_{0}=r_{0}\left(\left(p-p^{\prime}\right)^{2} / 4 m, \rho\right)$ определяется из второго соотношения в $(32)$, где $E=$ $\left(p-p^{\prime}\right)^{2} / 4 m, \rho \leqslant V_{\text {уд }}^{1 / 3}$, а $\phi$ лежит в плоскости рассеяния. Еще мы должны учесть ограничение, которое связано с тем, что объем, хотя и большой, но конечный. Поэтому прицельный параметр не может изменяться до бесконечности. Он ограничен для двух частиц величиной объема. В случае $N$ частиц он ограничен удельным объемом. Поэтому мы введем ограничение по $\rho$ вида $\rho \leqslant V_{\text {уд }}^{1 / 3}$. Фактор сжимаемости $Z$ равен

$$
Z=b \int_{0}^{V_{\text {уд }}^{1 / 3}}\left[\int_{0}^{\infty} \int_{0}^{2 \pi} \frac{p^{2} / 2 m d p^{3} d \phi}{e^{b\left(p^{2} / 2 m+u(p, \rho)-\mu\right)}-1} / \int_{0}^{\infty} \int_{0}^{2 \pi} \frac{d p^{3} d \phi}{e^{b\left(p^{2} / 2 m+u(p, \rho)-\mu\right)}-1}\right] \frac{\rho d \rho}{V_{\text {уд }}^{1 / 3}} .
$$

Однако, чтобы точно определить фазовые переходы газа или жидкости, необходимо существенно уточнить формулы (35), (36). Нужно отметить, что вероятность нахождения частицы в точке $x_{2}$ непостоянна на интервале от $r_{0}$ до бесконечности. Она, очевидно, пропорциональна времени пребывания частицы в некотором интервале, а это время обратно пропорционально скорости второй частицы относительно первой на этом интервале. Нетрудно убедиться в том, что эта вероятность равна

$$
F\left(x_{2}\right)=\frac{\left|\left[\frac{\left(p_{1}-p_{2}\right)^{2}}{m}-\frac{\rho\left(p_{2}-p_{1}\right)^{2}}{m\left(x_{2}-x_{1}\right)^{2}}-\Phi\left(x_{2}-x_{1}\right)\right]^{-1 / 2}-\left(\frac{\left(p_{1}-p_{2}\right)^{2}}{m}\right)^{-1 / 2}\right|}{\int_{r_{0}}^{\infty}\left|\left\{\left[\frac{\left(p_{1}-p_{2}\right)^{2}}{m}-\frac{\rho\left(p_{2}-p_{1}\right)^{2}}{m\left(x_{2}-x_{1}\right)^{2}}-\Phi\left(x_{2}-x_{1}\right)\right]^{-1 / 2}-\frac{\left|p_{1}-p_{2}\right|^{2}}{\sqrt{m}}\right\}^{-1 / 2}\right| d r} .
$$

Поскольку в результате интегрирования выражения

$$
\frac{F\left(x_{2}\right)}{e^{b\left(p_{2}^{2} / 2 m+u\left(p_{2}, \rho\right)-\mu\right)}-1}\left[\iint_{0}^{2 \pi}\left(e^{b\left(p_{2}^{2} / 2 m+u(p, \rho)-\mu\right)}-1\right)^{-1}\left|p_{2}\right|^{2} d p_{2} d \phi\right]^{-1},
$$

сначала по $x_{2}$, а затем по $d p$ получаем единицу, то распределения по $x_{2}$ и по $p_{2}$ независимы и равны произведению распределений по $x_{2}$ и по $p_{2}$ [25].

Учитывая это, мы получим поправку к (35), (36) и окончательный вид интегрального уравнения:

$$
\begin{array}{rl}
u(p, \rho)=2 & N \int_{0}^{\infty} \int_{0}^{2 \pi} \frac{\int_{r_{0}}^{\infty} \Phi(r)\left|\frac{\left(p-p^{\prime}\right)^{2}}{m}-\frac{1}{m} \frac{\left(p-p^{\prime}\right)^{2} \rho^{2}}{r^{2}}-\Phi(r)\right|^{-1 / 2} r^{2} d r}{\int_{r_{0}}^{\infty}\left|\left\{\frac{\left(p-p^{\prime}\right)^{2}}{m}-\frac{1}{m} \frac{\left(p-p^{\prime}\right)^{2} \rho^{2}}{r^{2}}-\Phi(r)\right\}^{-1 / 2}-\frac{\sqrt{m}}{\left|p-p^{\prime}\right|}\right| d r} \times \\
& \times \frac{1}{e^{b\left(\left(p^{\prime}\right)^{2} / 2 m+u\left(p^{\prime}, \rho\right)-\mu\right)}-1}\left|p^{\prime}\right|^{2} d\left|p^{\prime}\right| d \phi \mathrm{P}_{\rho}\left(\frac{\left(p-p^{\prime}\right)^{2}}{m}\right) \times \\
& \times\left\{\int_{0}^{\infty} \int_{0}^{2 \pi} \frac{1}{e^{b\left(p^{2} / 2 m+u(p, \rho)-\mu\right)}-1} p^{2} d p d \phi\right\}^{-1},
\end{array}
$$


где $b=a^{2} / V^{2 / 3} k T$. Это интегральное уравнение более точно определяет фазовый переход.

\section{0. ФАЗОВЫЕ ПЕРЕХОДЫ}

Исследование кластеризации и ее уменьшения с повышением температуры, а также теория перколяции - это отдельные быстроразвивающиеся направления моделирования динамики процесса образования и разрушения кластеров [21]. Они особенно актуальны при исследовании сложных молекул. С их помощью можно получить зависимость химического потенциала $\mu$ от температуры, если учесть соотношения из раздела 6. Если заданы температура (изотермический процесс) и химический по-

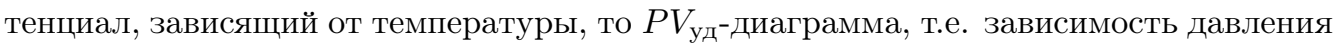
от объема, выражается следующим образом:

$$
P=\frac{R T Z}{V_{\text {уд }}} .
$$

Уравнение (39) является аналогом уравнения Ван-дер-Ваальса и других эмпирических соотношений. Оно определяет фазовые переходы.

Как автор неоднократно отмечал, распределение Гиббса пишут в виде $e^{-H(p, q) / k T}$, т.е. по существу без малого параметра в экспоненте. Но поскольку интеграл по $p, q$ от этого выражения равен числу частиц $N$, а $N \rightarrow \infty$, то малый параметр в экспоненте необходим. Поэтому если его не вводить, как это делал автор, то нужно полагать $k T \rightarrow \infty$, и малые температуры не рассматривать вообще или же считать, что константа Больцмана $k \rightarrow \infty$. Но это слишком непривычно (в отличие от случая, когда принято считать, что константа Планка стремится к нулю). В соответствии с этим нужно либо ввести параметр $b$ в потенциал Леннарда-Джонса при $\varepsilon \rightarrow \infty$, либо делить потенциал на безразмерный параметр $b k T$, что мы и сделаем. Тогда (32) превратится в

$$
\frac{\rho^{2}}{r_{0}^{2}}+\frac{(m / b k T) \Phi\left(r_{0}\right)}{\left(p-p^{\prime}\right)^{2}}=1
$$

и поэтому в предыдущих формулах мы должны заменить взаимодействие ЛеннардаДжонса $\Phi$ на $\Phi / b k T$, которое можно вновь обозначить через $\Phi$.

Сделав замену $b p^{2}=\bar{p}^{2}$ и $b p^{\prime 2}=\bar{p}^{\prime 2}$, мы избавляемся во всех соотношениях от малого параметра $b$ и приходим к окончательным формулам. Эта же замена позволяет исключить малый параметр и в интегральном уравнении для $u(p, \rho)$. При этом написанные новые соотношения будут соответствовать принятым у физиков, которые игнорируют безразмерный малый параметр $\alpha \sim b$, что и разумно, потому что в окончательных ответах он пропадает. При этом надо заменить прицельный параметр $\rho$ на $\bar{\rho} / \alpha$, тогда в (36) верхний предел $V_{\text {уд }}^{1 / 3}$ изменится на $\alpha V_{\text {уд }}^{1 / 3}$. Поэтому в окончательных формулах можно заменить $b$ на $\beta=1 / k T$. Однако для строгих доказательств малый параметр необходим. 


\section{1. СРАВНЕНИЕ С ЭКСПЕРИМЕНТАЛЬНЫМИ ДАННЫМИ}

В задаче термодинамики есть большой параметр - число частиц. Кроме того, имеется другой большой параметр - объем $V$. Мы рассматриваем удельный объем $V_{\text {уд }}=V / N$. Объем $V$ имеет размерность $3[26]$. В термодинамике также рассматриваются двумерная поверхность с большим параметром - числом частиц, и удельная поверхность. Очевидно, что между этими двумя целыми размерностями есть и другие нецелые размерности, которые получаются, когда удельный объем стремится к нулю. Иначе говоря, когда объем $V$ есть утолщенная поверхность, толщина которой $D \approx N^{\alpha-1 / 3}$ при $\alpha<1 / 3$ стремится к нулю при $N \rightarrow \infty$, при $\alpha=0$ получается поверхность $S$. Величина $\alpha$ определяет промежуточную размерность между 3 и 2 . Если речь идет о газе, то реально она определяется наличием нанопор в веществе и их объемом.

Этот эффект хорошо известен: пористая среда, состоящая на $30 \%$ из нанопор, вмещает под давлением количество газа, в 150 раз большее, чем пустая емкость того же объема [27]. Простое теоретическое объяснение этому содержится в приведенных формулах, так как максимальное число частиц зависит от фрактальной размерности и увеличивается при уменьшении размерности.

Максимальное число частиц в перенасыщенном газе (паре) в трехмерном объеме определяется из распределения типа Бозе-Эйнштейна. Для двумерного случая (поверхности) оно равно бесконечности. Однако для промежуточной фрактальной размерности, определенной нанопорами, оно конечно и стремится к бесконечности при размерности, стремящейся к 2. Если газ перенасыщен при фиксированной достаточно низкой температуре, то его минимальная сжимаемость $Z=P V / k_{\mathrm{B}} T N \cong 0.53$. Однако при этом может образовываться жидкость с нанопузырями, и размерность может резко упасть при увеличении давления, а следовательно, может упасть сжимаемость (сжимаемость в двумерной задаче для идеального газа с распределением типа Бозе-Эйнштейна равна нулю).

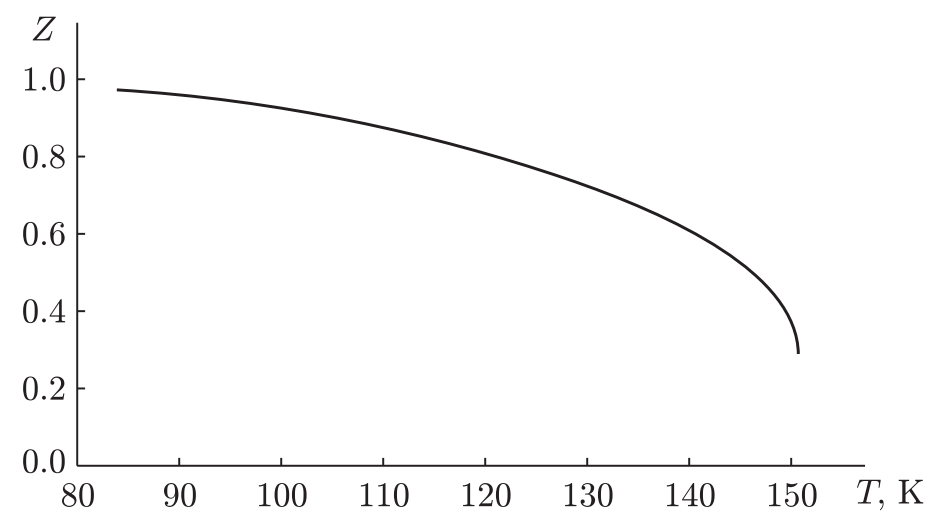

Рис. 2. Зависимость фактора сжимаемости от температуры. 
На рис. 2, 3 продемонстрированы термодинамические свойства насыщенного аргона.

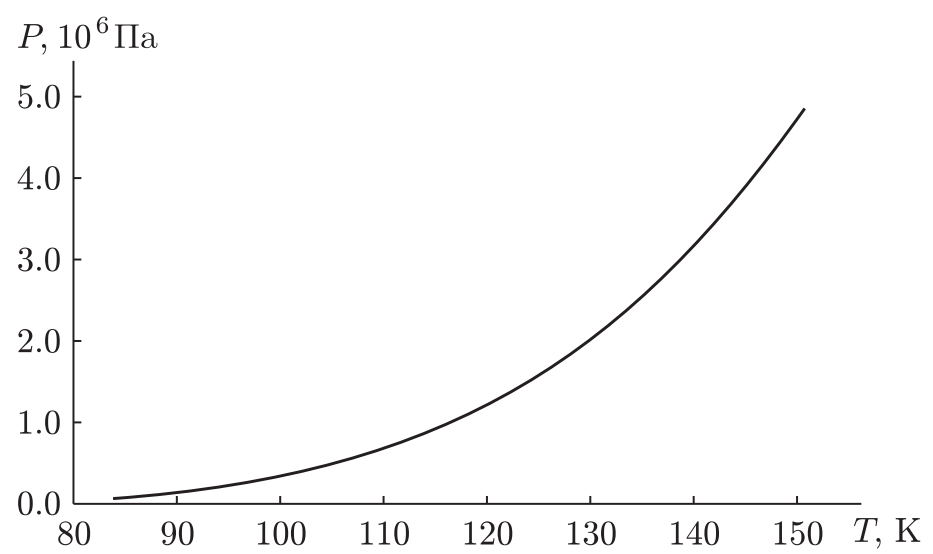

Рис. 3. Зависимость давления от температуры.

Если пар насыщенный, то при низкой температуре, как правило, число кластеров (димеров, тримеров) велико. Это уменьшает общее число частиц в объеме и увеличивает химический потенциал, а значит, увеличивается сжимаемость. При увеличении температуры число кластеров уменьшается и при некоторой температуре димеров становится меньше 7\% (критерий Кало). Тогда сжимаемость может упасть до 0.53. Но в силу того, что насыщенный газ находится в равновесии с жидкостью, которая содержит нанопузыри, размерность может достаточно резко упасть, и сжимаемость, например, для аргона уменьшится до 0.25. Частично может играть роль и включение взаимодействия при давлениях ниже точки минимума сжимаемости. Это взаимодействие является в основном притяжением при увеличении давления и отталкиванием при уменьшении $V_{\text {уд. }}$ В свою очередь, при уменьшении объема во фрактальной размерности нанопузырей возникает эффект кластеризации типа бозе-конденсата, поскольку $b \sim a^{2} / V^{2 / 3} T$, и уменьшение температуры и объема приводит к одному и тому же эффекту (см. раздел 6).

Таким образом, образование наноструктур в другой фазе (жидкости) играет существенную роль, как и образование кластеров в газе. Будем в дальнейшем называть такое образование наножидкостью размерности $2<q<3$, отвечающей величине сжимаемости.

Теперь перейдем к случаю постоянной температуры (см. рис. 4, где $T_{\mathrm{r}}$ и $P_{\mathrm{r}}-$ приведенные температура и давление соответственно [28]).

Пока сжимаемость $Z>0.523$, взаимодействие можно считать несущественным. При $Z<0.523$ включается взаимодействие (поскольку уменьшился объем), и оде-

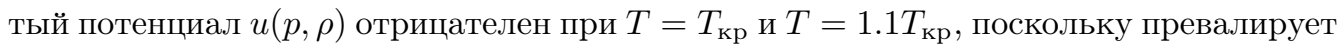

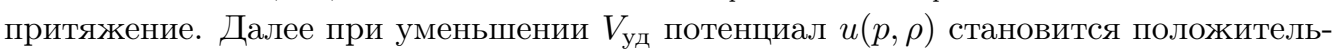




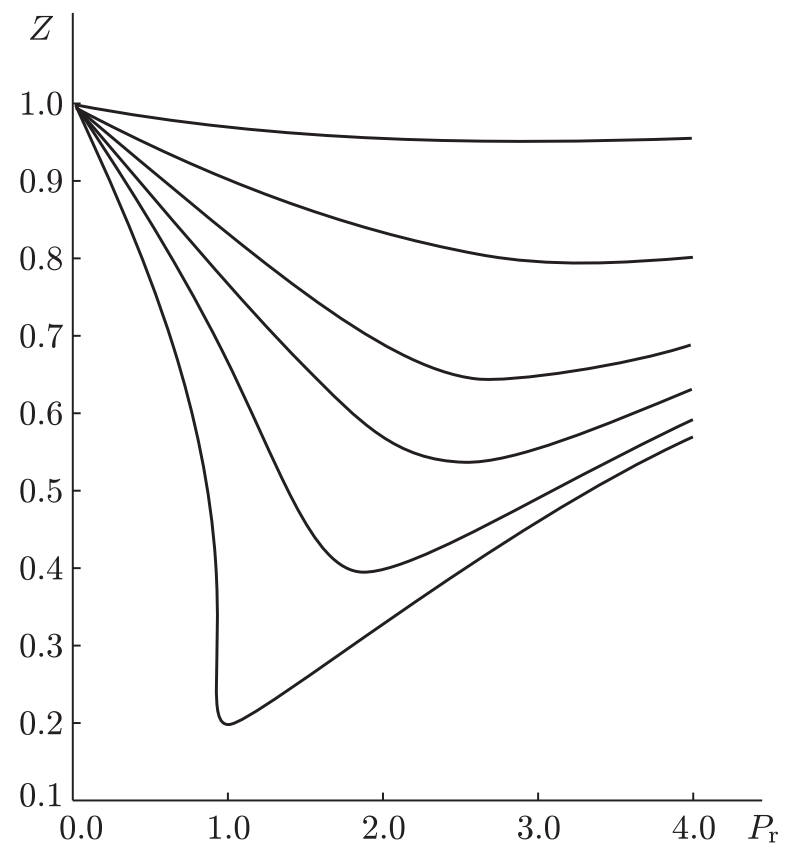

Рис. 4. Зависимость фактора сжимаемости от давления при различных температурах. Изотермы для десяти чистых газов (метан, этан, этилен, пропан, азот и др.).

ным, начинает проявлять себя отталкивание и сжимаемость увеличивается. Аналогичная ситуация и для других изотерм.

Процесс образования кластеров, описанный выше, увеличивает сжимаемость. Там, где взаимодействие несущественно, расхождение теории с экспериментом и между изотермами различных газов оказывается порядка 1-2\%. Включение взаимодействия увеличивает это расхождение.

Благодарности. Автор приносит глубокую благодарность А.K. Реброву, С. К. Гордееву и Б. В. Егорову за предоставление экспериментальных данных.

\section{Список литературы}

[1] В. П. Маслов, Матем. заметки, 83:5 (2008), 787-791.

[2] В. П. Маслов, ТМФ, 155:2 (2008), 312-316.

[3] В. П. Маслов, Матем. заметки, 78:6 (2005), 870-877.

[4] V.P. Maslov, Russ. J. Math. Phys., 14:1 (2007), 66-95.

[5] В. А. Алексеев, ЖЭТФ, 119:4 (2001), 700-709.

[6] В. П. Маслов, Докл. РАН, 392:6 (2003), 727-732.

[7] В. П. Маслов, Квантовая экономика, Наука, М., 2006.

[8] В.П. Маслов, Обозрение прикладной и промыиленной математики, 11:4 (2004), 679-732; 12:1 (2005), 3-40.

[9] Х. Гулд, Я. Тобочник, Компьютерное моделирование в физике, Мир, М., 1990. 
[10] В.В. Гуньков, Моделирование перколячионного кластера: методические указания $к$ лабораторному практикуму, ГОУ ОГУ, Оренбург, 2005.

[11] В. П. Маслов, В. Е. Назайкинский, Матем. заметки, 81:6 (2007), 879-892.

[12] А.М. Вершик, Функи. анализ и его прил., 30:2 (1996), 19-39.

[13] В. П. Маслов, В. Е. Назайкинский, Матем. заметки, 83:6 (2008), 880-898.

[14] В. П. Маслов, В. Е. Назайкинский, Матем. заметки, 84:1 (2008), 69-98.

[15] В.П. Маслов, Матем. заметки, 84:2 (2008), 312-317.

[16] А.И. Бурштейн, Молекулярнал физика, Наука, Новосибирск, 1986.

[17] Л. Д. Ландау, Е. М. Лифшиц, Статистическая физика, Наука, М., 1964.

[18] U. Kaiser, D. A. Muller, J. L. Grazul, Nature Materials, 1 (2002), 102-105.

[19] N. Lümmen, T. Kraska, Nanotechnology, 16 (2005), 2870-2877.

[20] K. Ohguchi, K. Yasuoka, M. Matsumoto, Progr. Theoret. Phys., 138, Suppl. (2000), $257-258$.

[21] Ю.Ю. Тарасевич, Перколяиия: теория, приложения, алгоритмы, Едиториал УРСС, M., 2002.

[22] В. П. Маслов, Матем. заметки, 83:6 (2008), 864-879.

[23] Л. Д. Ландау, Е. М. Лифшиц, Теоретическая физика. Т. І. Механика, Физматлит, М., 1958.

[24] Ю. Г. Павленко, Лекиии по теоретической механике, Физматлит, М., 2002.

[25] В. П. Маслов, ТМФ, 157:1 (2008), 149-153.

[26] В.П. Маслов, Матем. заметки, 84:1 (2008), 149-152.

[27] С. К. Гордеев, Исследование температурной зависимости адсорбиии этана и метана углеродными нанопористыми материалами, Отчет "B.R.F. - инновации и технологии", 2003.

[28] Citizendium, Compressibility factor (gases), http://en.citizendium.org/wiki/ Compressibility factor. 